\title{
BAVIEERE
}

\section{LA CONVENTION DE GENÈVE}

par le Dr G. Lueder.

La littérature du Droit des gens s'est enrichie, l'an dernier, d'un nouvel ouvrage dù à lá plume du docteur C. Lueder, professeur de droit à l'université d'Erlangen. On connaît l'origine de ce travail. A l'occasion de l'exposition de Vienne en 1873, S. M. l'Impératrice d'Allemagne, un des plus fermes soutiens de l'cuvire de la Croix rouge, avait institué un prix de 2,000 thalers, à décerner à la meilleure étude sur la Convention de Genève. Le mémoire du docleur Lueder, soumis inachevé à l'appréciation du Jury international, fut néanmoins jugé supérieur aux autres écrits envoyés. Fventuellemeut désigné pour être couronné, il fut rendu à son auteur qui le compléta à l'entière satisfaction des juges. Le jury, par une décision unanime, lui décerna alors la récompense offerte par l'Tmpératrice.

A l'époque où fut ouvert ce concours, le Bulletin du Comité international en a donné le programme. (Bulletin no 17, t. V, p. 18). Le travail devait fournir un « aperçu historique du développement de la Convention de Genève, depuis son origine jusqu'à ce jour; donner un exposé des expériences auxquelles l'application et la mise en ceuvre de la Convention ont donné lieu; contenir enfin les propositions relatives à l'extension et au développement ultérieur qu'il s'agirait d'accorder à la Convention, ainsi qu'aux modifications qu'il serait urgent d'y apporter.»

Le plan se trouvait ainsi tracé, et l'ouvrage, 'tout naturellement divisé en deux parties; un livre premier pour l'histoire de la Convention de Genève, un livre second pour l'examen critique de ce traité, pour l'exposé des expériences faites dans son application, pour les améliorations qu'il serait désirable d'y voir introduire.

Qu'est-ce que la Convention de Genève: un instrument diplomatique par lequel la plupart des Etats européens se sont engagés à observer, dans la conduite de la guerre, certaines règles destinées 
à améliorer la position des victimes. Cette préoccupation du sort des blessés et des malades n'a pas surgi brusquement à l'époque du congrès de Genève de 1864. Il y a longtemps que les guerres ont des conséquences fâcheuses pour la santé de milliers d'individus, longtemps aussi que la vue de leurs souffrances a inspiré à leurs chefs le désir d'en procurer l'allégement. Il serait donc injuste et puéril de faire honneur à notre temps de l'éclosion première de ces sentiments d'humanité. Le devoir de l'historien qui étudie la réalisation d'une idée est de remonter à la naissance de cette idée et d'en suivre le développement. C'est ici que l'investigation patiente et minutieuse de la critique allemande a pu se donner libre cąrrière. De l'année 1581 à l'année 1864, pas un traité relatif à l'échange et à la rançon des prisonniers de guerre, aux capitulations de troupes, forteresses, etc., au soin des malades ou des blessés laissés en pays étranger ou ennemi, à la protection des établissements thermaux et des blessés qui s'y faisaient traiter, pas une convention de ce genre qui ait échappé à ces infatigables chercheurs. Le docteur Lueder a surtout mis à contribution les remarquables travaux de Gurlt, qui ne cite pas moins de 291 traités intervenus dans cette période d'environ 280 années. Une des plus curieuses el des plus importantes de ces conventions est celle conclue entre les rois de France et de Prusse, à Brandebourg, "le septième du mois de septembre de l'année 1759 ", et dans laquelle se lisent les dispositions suivantes :

* Art. xxiri. Les aumôniers, ministres, médecins, chirurgiens, apothicaires, etc., ne seront point sujets à être faits prisonniers de guerre.

( Art. xxvir. On prendra soin des blessés de part et d'autre; on paiera les médicaments et leur nourriture, les frais seront restitués de part et d'autre; ceux qui auront été faits prisonniers aussi bien que ceux qui ne le seraient pas seront renvoyés sous la sauvegarde et protection des généraux, avec liberté d'ètre transportés suivant la plus grande commodité et convenance, et par le plus court chemin, à condition, toutefois, que ceux qui auront été faits prisonniers ne serviront pas qu'ils ne soient échangés ou rançonnés.

"Art. xxvirr. Les malades de part et d'autre ne seront pas faits prisonniers; ils pourront rester en sûreté dans les hôpitaux où il sera libre à chacune des parties belligérantes de leur laisser une 
garde, laquelle ainsi que les malades seront renvoyés sous des passeports respectifs des généraux, par le plus court" chemin, et sans pouvoir être troublés ni arrêtés. Il en sera de même des aumôniers, médecins, chirurgiens, apothicaires, garçons infirmiers, servants ou autres personnes propres pour le service des malades, lesquels ne pourront être faits prisomniers et seront pareillement renvoyés."

Ne croirait-on pas lire la Convention de Genève rédigée en français du milieu du $18^{\circ}$ siècle ? N'est-on pas en droit de conclure de pareils documents que la pensée mère de la Convention était réalisée depuis longtemps, dans des traités auxquels il ne manquait que le caractère définitif et international ?

Et pourtant ni les guerres de la première république française et de l'empire, ni même soixante ans après, la longue lutte dont la Crimée fut le théâtre lointain, n'ont amené la conclusion d'un traité permanent. La guerre d'Italie de 1859 attira davantage l'attention des philanthropes, et, dans les deux années qui suivirent, trois hommes de nationalités différentes firent presque simultanément appel à l'opinion publique européenne en faveur des victimes des combats. Ce sont, par ordre de date, le docteur Palasciano, de Naples (avril 1861), M. Arrault, de Paris (juin 1861), et M. H. Dunant, de Genève (1862). M. Palasciano demandait la reconnaissance du principe de neutralité des blessés et des malades, et l'augmentation du personnel sanitaire en temps de guerre; M. Arrault voulait la neutralisation des médecins militaires et des ambulances, ainsi que celle des porteurs de blessés et des places de pansement; M. Dunant proposait de créer de vastes associations pour secourir les malades et les blessés. Toutes ces idées, également nobles, également fécondes, méritent à leurs promoteurs une égale reconnaissance de la postérité. En un pareil sujet, la question de priorité n'a guère d'importance que pour les persomnes intéressées. Aussi, quand le docteur Palasciano réclame pour Naples, dans ses Archives de chirurgie pratique (no du 29 février 1876), la priorité de l'idée de neutralisation des blessés, nul ne lui cherchera-t-il querelle. Mais où la défense de son droit l'entraine trop loin, c'est lorsqu'il ajoute: "Que le professeur Lueder se domne la peine de relire mon dis" cour's à l'académie Pontaniana, et de le confronter avec le Sou- venir de Solferino, l'ouvrage à grand retentissement de M. Dunant, 
" et puis qu'il dise loyalement d'où est partie la Convention, si " c'est de Naples ou de Genève. » Le docteur Lueder, qui a sans doute lu le discours du docteur napolitain, pourrait lui répondre et lui répond par avance dans sa discussion historique: la Conven. tion porte le nom de Genève, et non celui de Naples, par la raison qu'elle est l'œuvre du congrès de Genève, lequel est issu des décisions de la conférence de Genève de 1863, qui était elle-même le produit de l'activité de quelques membres d'une société philanthropique genevoise.

A son tour, notre auteur nous paraît céder trop facilement au plaisir de châtier la vanité française, manquer en tout cas aux préceptes de la galanterie, lorsqu'il relève si vertement " l'ignorance crasse " dont a fait preuve Madame George Sand, en célébrant Arrault comme le promoteur de la Convention de Genève. Le soin jaloux avec lequel il défend la gloire de M. Dunant et de la Société d'utilité publique genevoise, paraît un peu trop inspiré par le désir de bien constater que la Convention de Genève ne doit pas son origine à une idée française.

Des préoccupations du même genre se font jour à plusieurs reprises dans le mémoire du docteur Lueder. M. Dunant, dans son Souvenir de Solferino, ayant rapporté quelques traits de barbarie imputables à des soldats autrichiens, le professeur d'Erlangen prend vivement la défense de l'armée autrichienne, et reproche $\dot{a}$ la France ses turcos algériens. Il aurait préféré, dit-il, ne pas revenir sur ces faits déjà anciens, mais il s'y est vu contraint dans l'intérêt de la justice et de la vérité. Bien des passions ne se satisfont-elles pas dans le monde au nom de la justice et de la vérité? Notre auteur affectionne tout particulièrement la figure de la prétérition. Il a une manière de dire : ce n'est pas ici le lieu d'examiner si..., laissons de cóté la question de savoir si..., qui lui permet d'émettre sous une forme dubitative des jugements que l'on sent très-arrêtés dans son esprit. Ainsi, après avoir pesé les diverses influences qui ont agi sur les délibérations du congrès de Genève, et avoir constaté la prépondérance de l'influence française, soutenue par les délégués suisses, il ajoute : "Laissons de côté la question de savoir si ces expressions et ces encouragements peuvent être approuvés par le penseur qui va au fond des choses, qui sait distinguer l'apparence de la réalité, el si l'influence prépondérante 
dans le congrès a bien été accordée à ceux qui avaient qualité pour l'exercer. "On aperçoit bien qui, dans l'opinion de M. Lueder, avait qualité pour exercer une influence, mais il serait plus digne d'une critique vraiment sérieuse et impartiale, de dire en quoi telle ou telle influence a été fâcheuse pour la Convention; quelle faute, quelle erreur elle a fait commettre; au lieu de se borner à insinuer que, d'un còté, s'exerçait une autorité usurpée, et que, de l'autre côté seulement, se trouvaient les qualités indispensables pour discuter avec fruit le traité soumis aux délibérations du congrès. Nous pensons que de telles insinuations n'ajoutent rien à l'autorité scientifique très-réelle de cet ouvrage, mais qu'elles pourront lui òter de son autorité morale, et nuire à son succès hors d'Allemagne.

Il nous est impossible de suivre l'auteur dans les développements historiques qui forment le premier livre de son mémoire. Il nous fait assister successivement à l'émotion suscitée par le Souvenir de Solferino, aux décisions et démarches de la Société genevoise d'utilité publique pour arriver à la réalisation des idées de M. H. Dunant et de ses émules; il nous conduit à la conférence de Genève d'octobre 1863 , au congrès international tenu dans la même ville, du 8 au 22 août 1864. De Genève, nous passons ensuite à Berlin, oủ se réunit, au printemps de 1867, une conférence sanitaire militaire; à Wurzbonrg, où se rassemblent les sociétés de secours allemandes, le 22 aout 1867; à Paris, où se tient une assemblée internationale du 26 au 31 août 1867; il nous ramène enfin à Genève pour le deuxième congrès international du 5 au 20 octobre 1868 , lequel rédigea les Articles additionnels à la Convention de Genève. Gette première partie se termine par l'histoire du développement ultérieur de l'idée mère de la Convention, depuis le congrès de 1868 jusqu'à ces derniers temps, et par le compte rendu de la conférence de Bruxelles du 27 juillet au 27 aoùt 1874. Elle nous a paru traitée avec une exactitude rigoureuse, un soin des détails presque minutieux; pas un nom, pas une date ne sont oubliés. Si nous nous reportons au programme du concours, nous dirons que l'aperçu historique demandé est devenu, dans le travail de M. Lueder, une tue très-complète et très-détaillée. Il ne sera plus permis désormais d'écrire sur la Convention de Genève sans avoir soigneusement feuilleté l'ouvrage du professeur d'Erlangen. 
Avec le livre deuxième, nous abordons la partie dogmatique de l'œuvre, l'examen critique de la Convention envisagée comme texte du Droit des gens, et la discussion de ces deux questions : faut-il maintenir la Convention de Genève? sous quelle forme doit-elle être maintenue?

Le traité du 22 août 1864 compte des admirateurs et des détracteurs également passionnés. Les uns y voient le triomphe définitif des idées humanitaires sur la force brutale, l'aurore de la paix universelle; d'autres affirment qu'il n'a apporté aucune amélioration réelle au sort des blessés et des malades; que, bien plus tòt, il entrave les mesures d'humanité que les chefs militaires seraient individuellement disposés à adopter. La vérité est à égale distance de ces deux manières de voir extrêmes.

La Convention de Genève, soumise à l'épreuve de la guerre de 1866, et surtout de la grande lutte de 1870-71, a montré qu'elle pouvait rendre de grands services; mais en même temps les défectuosilés se sont fait jour, et la nécessité d'une révision s'est affirmée aux yeux de tous les partisans éclairés de ce traité. Les violations de la Convention, que chacun des deux partis a vivement reprochées à son adversaire, ne sont pas suffisantes pour la déclarer mauvaise et inexécutable. Quand elle sera mieux connue et plus généralement appréciée des troupes, elle sera mieux pratiquée et moins souvent violée. Aussi les amis de la Convention doivent-ils désirer ardemment qu'elle puisse recevoir toutes les améliorations nécessaires pour lui faire trouver grâce auprès de ceux qui en contestent aujourd'hui l'utilité. Une révision du traité de 1864 devra s'appuyer sur une base vraiment pratique, non sur les utopies ou les rêves des idéologues. Cette base solide repose tout entière sur ce principe que le but d'humanité ne doit être pris en considération qu'autant que le but militaire le permet. C'est à la lueur de ce flambeau que le docteur Lueder examine tous les articles de la Convention de Genève, et ceux proposés par le congrès de 1868 sous le nom d'Articles additionnels. Le suivre sur ce terrain nous entrainerait beaucoup au-delà des limites qui nous sont imposées. Parmi les critiques de fond formulées par l'auteur, il en est plusieurs qui nous paraissent justifiées; sur d'autres points, nous avons des réserves à faire, et lorsque l'occasion s'en présenlera, nous les ferons connaître. 


\section{5}

L'insistance avec laquelle le professeur laurẻat affirme la nécessité de subordonner le but d'humanité de la Convention de Genève au butmalériel de laguerre, nous avait fait craindre de trouver dans sa rédaction trop de concessions faites à ce tyran redoutable qui s'appelle : les exigences militaires. Aussi, notre surprise a-t-elle été fort agréable, quand nous avons vu l'honorable professeur s'élever contre l'idée de certains écrivains qui vondraient conserver à l'armée occupante un droit de prise (Beuterecht) sur le matériel des établissements fixes, au lieu de limiter, sur ce point, le pouvoir du vainqueur à un droit d'usage. Cette dernière disposition, qu'il accepte, jointe à celle qui prohibe la rétention du matériel des établissements mobiles, doit être signalée comme un indice de l'esprit d'équité et de justice dont le docteur Lueder a cherché à s'inspirer dans la rédaction de son projet.

Une autre proposition de notre auteur, qui nous paraît aussi fort acceptable, est celle contenue dans son article $2^{\text {me }}$. Cet article impose à l'armée qui occupe le champ de bataille, l'obligation de le surveiller, de faire respecter les blessés et les morts contre le brigandage et les mauvais traitements, de constater l'identité des cadavres de faire procéder àleurinhumation, de faire parvenir aussitòt que possible à la partie adverse des listes exactes des morts, des blessés, et des prisonniers tombés entre ses mains. Cette dernière obligation suppose l'existence sur chaque soldat d'une pièce établissant son identite.

En ce qui concerne la forme actuelle de la Convention, nous sommes entièrement de l'avis du professeur d'Erlangen quand il dit qu'une loi du droit des gens, comme la Convention de Genève, doit avant tout être simple, claire, précise, non susceptible d'interprétations diverses. Il est certain qu'à ce point de vue, le traité de 1864 laisse à désirer, surtout si on le rapproche des Articles additionnels de 1868. A ce propos, nous ferons observer qu'on a tort d'assimiler complétement et de réunir en un seul tout les Articles additionnels et la Convention, car l'œuvre du Congrès de 1868 n'a pas reçu la même consécration que la Convention de Genève, adoptée par toutes les puissances européennes. Ce qui demeure vrai, c'est qu'il était impossible de créer d'emblée une œuvre qui satisfît tout le monde, et qui à l'expérience, fût proclamée parfaite. Les différents auteurs qui ont écrit sur la Convention de 
Genève ont recherché la cause des imperfections de ce trailé, et, chose facile à prévoir, les médecins militaires ont déclaré qu'elles provenaient de ce que l'élément médical militaire était trop faiblement représenté au Congrès, tandis que les jurisconsultes ont déploré l'absence d'hommes experts dans la connaissance du droit des gens. Cetle dernière opinion est, en particulier, celle du professeur d'Erlangen. Nous la croyons fondée dans une certaine mesure; mais nous ne pouvons nous associer à la critique des mots "neutre et "neutralité " présentée par notre auteur, et qui a été formulée aussi par le représentant del'Allemagneà la conférence de Bruxelles. Sans doute, la neutralité du personnel sanitaire ne peut pas être assimilée à la neutralité d'un Etat non belligérant; mais les mots ne peuvent-ils pas s'employer également bien dans des sens un peu différents? L'essentiel n'est-il pas que ces mots présentent uneidée claire et facile à saisir? Et si en outre, ces mols sont entrés dans la compréhension générale avec un sens exact, n'est-ce pas l'important? Quels inconvénients sont résultés de l'emploi de ces mots " neutre et neutralité ? On n'en indique ancun. Nous avons bien l'affirmation du docteur Löffler, que le texte de la Convention de 1864 serait beaucoup plus pratique si le terme "neutre " n'existait pas, parce qu'on serait alors forcé d'énoncer bien exactement lidée que l'on enveloppe dans ce mot. Mais on perd de vue, nous semble-t-il, ce fait que la Convention s'adresse non-seulement aux autorités et aux savants, mais aussi aux masses; qu'il y a certains mots qui représentent vivement aux yeux des populations tout un ensemble d'idées, et qu'ure fois ces mots acceptés et compris, il y a plus d'inconvénients que d'avantages à les abandonner. Or, il nous paraît démontré jusqu'à l'évidence que tout ce qui est revêtu du symbole de la Croix rouge est associé, dans l'esprit des populations, à l'idée d'un domaine à part, d'un terrain neutre, celui de l'humanité, de la charité, enclavé, pour ainsi dire, dans celui de la guerre, de la lutte, de l'inimitié et de la violence. C'est dans ce sens que le personnel et le matériel sanitaires sont mis à part, soustraits au droit de la guerre; c'est pour cela que la Convention de Genève doit, à notre sens, poser comme art. $1^{\text {er }}$ ce principe fondamental : " Le personnel el le matériel du service sanitaire des armées sont déclarés neutres, et doivent, comme tels, ètre respectés et protégés par les belligérants. "Aucun des substantifs pro- 
posés pour remplacer celui de neutralité ne rend aussi bien l'idée qu'il doit exprimer. Nous ne pouvons accepter, comme équivalent, le terme d'inciolabilité proposé par le représentant de l'Allemagne. Ce mol ne tient compte que du côté passif du principe de la Convention. La nutralité implique non-seulement un droit, un bénéfice, celui d'inviolabilité, mais aussi un ensemble d'obligations à remplir, de condilions à observer, sans lesquelles le béneffe disparaîtra, sera refusé. Si, d'autre part, la rédaction de la Convention recevait une nouvelle forme claire, précise, sans équivoques, nous croyons qu'il n'y aurait aucun inconvénient à conserver les mots de " neutralité " et de "neutre, "mots auxquels l'instinct populaire ne s'est pas trompé, et qui sont bien entrés dans le domaine public.

Quoi qu'il en soit de ces divergences d'opinions, nous ne pouvons que remercier, en terminant, le docteur Lueder du beau livre dont il a doté la littérature du droit des gens, et spécialement celle de la Convention de Genève. Ce volume doit avoir sa place dans la bibliothèque de lous les amis de la Croix ronge, dont il a arborć lui-même le symbole. Une traduction en langue française, qui s'élabore par les soins du Comité international, étendra pro. chainement le cercle de ses lecteurs; nous ne doutons pas qu'il n'obtienne aussi à l'étranger l'accueil distingué qui lui a été fait en Allemagne, et qui a pleinement ratifié en sa faveur, la décision du Jury international.

E. Onier.

\section{BELGIQUE}

CONFÉRENGE INTERNATIONALE JE LA CROIX ROUGE a bRUXELLES EN 1876

Le Comité central belge a adressé à tous les conités centratix des autres pays, la circulaire suivante: ${ }^{1}$

1 Voir la note p. 76. 